\title{
Radiation Inhibits Lymph Drainage in an Acquired Lymphedema Mouse Hindlimb Model
}

\author{
Zhe Wang, MD, ${ }^{1-3,{ }^{*}}$ Kun Yung Kim, MD, ${ }^{4, *}$ Sung Hwan Yoon, MS, ${ }^{1,2}$ Jung-Hoon Park, PhD, ${ }^{1,2,5}$ \\ Joonmyeong Choi, PhD, ${ }^{1,2,6}$ Nader Bakheet, MD, ${ }^{1,2}$ Hong Tao Hu, MD, 1,2,7 Jorge E. Lopera, MD, 8 \\ Ho-Young Song, MD, PhD, ${ }^{1,2, \dagger}$ and Jae Yong Jeon, MD, $\mathrm{PhD}^{9, \uparrow}$
}

\begin{abstract}
Background: Radiation therapy has been applied to prolong the duration of lymphedema. This study aimed to evaluate the effect of radiation on the development of lymphedema in a mouse hindlimb model.

Methods and Results: A total of $24 \mathrm{Balb} / \mathrm{c}$ mice underwent the right popliteal lymph node excision and the afferent and efferent lymphatics blockage. The radiation group $(n=12)$ received a single 20 Gy radiation 1 day before surgery in the right hindlimb of each mouse, whereas the control group $(n=12)$ only received surgery without radiation. The right hindpaw thickness of each mouse was measured twice a week for 4 weeks. Fluorescence microscopy images using fluorescein isothiocyanate-dextran tracer were obtained once weekly. Immunohistochemical (IHC) staining images using anti-lymphatic vessel endothelial hyaluronan receptor-1 (anti-LYVE-1) were obtained at 4 weeks after surgery. The radiation group showed significant increase in the thickness of the right hind paws from 0.5 to 2 weeks compared with the control group. As for fluorescence lymphography, the radiation group showed a lower number of regenerated lymphatics and more congestion of tracers in the operated limb at the surgery sites at 1,2,3, and 4 weeks after surgery. For the IHC analysis, the radiation group showed a lower number of regenerated lymphatics per high-power field at the surgery site than the control group.

Conclusion: Radiation therapy transiently aggravated the extent of lymphedema by inhibiting regenerated lymphatics in a mouse hindlimb model. However, it did not prolong the duration of lymphedema because the cutaneous interstitial flow contributes to the lymphatic fluid clearance.
\end{abstract}

Keywords: experimental lymphedema, radiation, lymphangiogenesis, lymph drainage

\section{Introduction}

$\mathbf{L}$ YMPHEDEMA IS A PATHOPHYSIOLOGIC PROCESS characterized by the accumulation of protein-rich fluid in the interstitial space, adipose deposition, and tissue fibrosis resulting from injury, infection, obstruction, or congenital defects in the lymphatic system. Globally, the most common cause of lymphedema is filariasis that occurs owing to infection by the nematode Wuchereria bancrofti. ${ }^{1}$ In the developed world, majority of lymphedema cases are secondary and caused by malignancy or its treatment. ${ }^{2-6}$ Lymphedema is a chronic condition that can be managed; however, it is generally not cured. ${ }^{7}$ As the treatment of chronic lymphedema remains challenging, the preclinical studies of the

Departments of ${ }^{1}$ Radiology and ${ }^{2}$ Research Institute of Radiology, Asan Medical Center, University of Ulsan College of Medicine, Seoul, Republic of Korea.

${ }^{3}$ Department of Radiology, Tianjin Medical University General Hospital, Heping, P.R. China.

${ }^{4}$ Department of Radiology, Research Institute of Clinical Medicine, Chonbuk National University-Biomedical Research Institute, Chonbuk National University Hospital, Jeonju-si, Republic of Korea.

${ }_{5}^{5}$ Department of Biomedical Engineering Research Center, Asan Medical Center, University of Ulsan College of Medicine, Seoul, Republic of Korea.

${ }^{6}$ Department of Mechanical and Aerospace Engineering, Seoul National University, Seoul, Republic of Korea.

${ }^{7}$ Department of Minimal-Invasive Intervention, The Affiliated Cancer Hospital of Zhengzhou University, Zhengzhou, China.

${ }^{8}$ Department of Radiology, UT Health Science Center at San Antonio, San Antonio, Texas.

${ }^{9}$ Departement of Rehabilitation Medicine, Asan Medical Center, University of Ulsan College of Medicine, Seoul, Republic of Korea.

*These authors contributed equally to this work and are co-first authors.

${ }^{\dagger}$ These authors contributed equally to this work and are co-corresponding authors. 
underlying pathophysiology and therapeutic interventions of lymphedema are urgently warranted. A reliable and reproducible lymphedema animal model is quite essential for such studies. The mouse tail model, by blocking lymphatic collateral vessels located at both sides of a rodent tail, was used to clarify the underlying mechanisms of lymphedema development in most recent studies. ${ }^{8-10}$ Tail model has the advantages of low cost, easy handling, and its effectiveness in interpreting the molecular mechanisms of lymphedema; however, as the pattern of lymphatic flow and regeneration in rodent tail is different from that of humans, there is a necessity of an animal model that mimics the secondary lymphedema of humans.

A number of hindlimb models in rodents, ${ }^{11-16}$ which are more complex to create but physiologically and anatomically more similar to human postsurgical lymphedema, have been reported with various techniques. Most of previous studies using a hindlimb model reported gain of secondary lymphedema in the affected limb within 1 week after model creation surgery. However, the improvement of lymphedema usually occurred within 2-3 weeks because of wound healing, accompanied by lymphatic channel regeneration. To prolong the duration of secondary lymphedema, radiation therapy was applied before or after surgery. ${ }^{13,14}$ Theoretically, radiation therapy is expected to damage the healing competence of tissue and thus augment the duration and degree of secondary lymphedema. However, no comparative study has been conducted to investigate whether radiation therapy truly affects the results of the experimental secondary hindlimb lymphedema.

In this study, we hypothesized that conducting a surgery to create lymphedema preceded by radiation therapy may augment secondary lymphedema by inhibiting lymphangiogenesis and hindering lymphatic channel regeneration compared with conducting a surgery without radiation therapy. Thus, the purpose of this study was to evaluate the effect of radiation therapy on the duration and degree of secondary lymphedema in an experimental hindlimb lymphedema model.

\section{Materials and Methods}

This study was approved by the committee for animal research at our institution and conformed to the U.S. National Institutes of Health Guidelines on the care and use of laboratory animals.

\section{A lymphedema mouse hindlimb model}

Hindlimb lymphedema was created in 10-week-old female Balb/c mice (Orient Bio, Seong-nam, Korea) as described previously, ${ }^{14}$ but with a modification. We did not inject an indicative blue dye to avoid its toxic effect. In brief, the mice were anesthetized with intramuscular injection of $50 \mathrm{mg} / \mathrm{kg}$ zolazepam and tiletamine (Zoletil 50; Virbac, Carros, France) and $10 \mathrm{mg} / \mathrm{kg}$ xylazine (Rompun; Bayer HealthCare, Leverkusen, Germany). The right-side inguinal skin was circumferentially incised, followed by location of the popliteal lymph node (Fig. 1a) and complete resection with the surrounding adipose tissue. A hole at the facial layer was noted after the lymph node resection (Fig. 1b). We cauterized the inner wall of the hole using a Bovie cautery to block both afferent and efferent lymphatics. Finally, both the ends of the skin wound were directly sutured with 5-0 nylon sutures. This surgical procedure took $\sim 30$ minutes by a single operator.

\section{Mouse limb irradiation}

The irradiation was performed a day before surgery for the radiation group. The mice $(n=12)$ were anesthetized with zolazepam-tiletamine, and then the right inguinal areas were exposed and irradiated with $20 \mathrm{~Gy}$ in a single dose. The ionizing radiation was emitted from the X-RAD 320 machine (Precision X-Rad, North Branford, CT) at a dose rate of 4.12 $\mathrm{Gy} / \mathrm{min}(150 \mathrm{kVp}, 20 \mathrm{~mA})$. The rest of the mouse body was shielded from radiation by using lead blocks. The control group $(n=12)$ mice received anesthetic and were placed in the irradiator, but they received no radiation. All mice were maintained in a temperature-controlled room $\left(22 \pm 2^{\circ} \mathrm{C}\right)$ and supplied with food and water ad libitum. After the respective observation periods, the mice were killed by carbon dioxide asphyxiation.

\section{Hindpaw thickness measurements}

Digital images of mouse hind paws were collected twice a week. Canon D550 camera was mounted on a tripod for imaging mouse paws. Hindpaw thickness was measured in standardized locations ( $2 \mathrm{~mm}$ proximal to the tarsal joint $)^{17}$ from these digital images using ImageJ imaging software (U.S. National Institutes of Health, Bethesda, MD).

\section{Fluorescence lymphography}

Fluorescence lymphography, which was performed once weekly after surgery for 4 weeks, was used to evaluate the distribution of regenerated lymphatic vasculature and lymphatic flow in the surrounding tissue. Lysine-fixable fluorescein isothiocyanate-dextran of $2000 \mathrm{kDa}$ (Molecular Probes) was used as a tracer. Tracer $(5 \mu \mathrm{L})$ was injected intradermally into the right hind palms for in vivo lymphatic imaging, with a gentle massage of the footpad for better
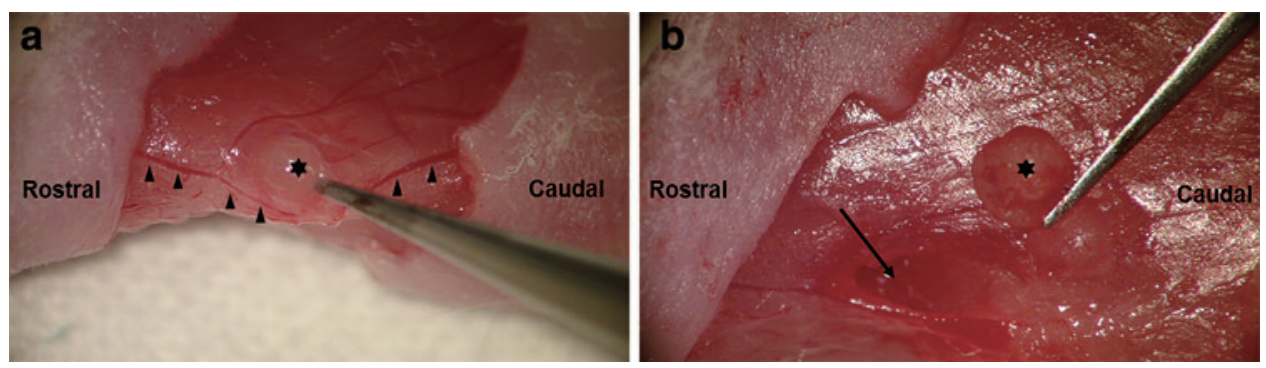

FIG. 1. (a) Arrow heads indicate the ischiatic vein. Asterisk indicates the popliteal lymph node. (b) Arrow indicates the hole formed by the tissue defect after the popliteal lymph node resection. Color images are available online. 


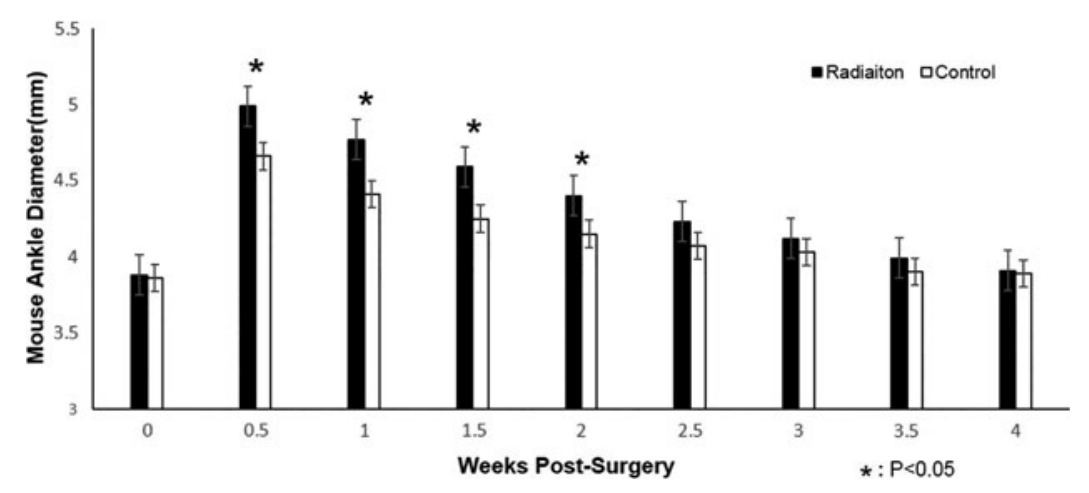

FIG. 2. Changes in the mice hindpaw thickness after surgery. The radiation group showed a significant augment compared with the control group during 2 weeks after surgery.

distribution. Digital images were taken immediately with a microscope-based multispectral imaging system for examination of the uptake of fluorescence and lymphatic pattern. In brief, a Nuance ${ }^{\mathrm{TM}}$ spectral imaging system (PerkinElmer, Inc., Hopkinton, MA) was mounted onto a conventional fluorescence microscope equipped with a filter cube comprising a standard excitation filter and dichroic mirror and a long-pass emission filter.

\section{Immunohistochemical staining}

After 4 weeks of surgery, all mice were killed using carbon dioxide asphyxiation, and then the right hindlimb of each mouse was cut off from the body. We collected the sample including the surgical area for immunohistochemical (IHC) staining. The surgical area and the range of sample collection are given in Figure 4a. The tissue was immersed in paraformaldehyde perfusion fixative (Electron Microscopy Sciences, Hatfield, PA) overnight at $4^{\circ} \mathrm{C}$. After decalcification, the samples were embedded in paraffin and sectioned into $5 \mu \mathrm{m}$ slices. After deparaffinization, rehydration, and blocking, the anti-lymphatic vessel endothelial hyaluronan receptor-1 (antiLYVE-1) polyclonal antibody (Abcam, Cambridge, United Kingdom) was used for IHC staining of lymphatic vessels at a dilution of $1: 100$.

The IHC images taken from the tissue of $5 \mathrm{~mm}$ distal to the surgery site were analyzed by two experienced observers, independently. Five random areas were selected with the same scale per high-power field (HPF) at $200 \times$ magnification. The number of lymphatic vessels stained by anti-LYVE1 in each area was counted, and the mean value of the number of lymphatic vessels from the five areas was calculated.

\section{Statistical analysis}

Statistical analysis was performed using SPSS version 22.0 (SPSS, Inc., Chicago, IL). The comparison of hindpaw thickness between the two groups was performed using the Student's $t$-test. The mean number of vessels per HPF was compared using the Student's $t$-test. All $p$-values were twosided with statistical significance evaluated at the 0.05 alpha level, and $95 \%$ confidence intervals were constructed to assess the precision of the obtained estimates.

\section{Results}

\section{Hindpaw thickness measurements}

During the observation period, all mice developed an acute lymphedema immediately after surgery. In the radiation group, only one mouse developed hindlimb necrosis. The data of this mouse were excluded from this study.

The thickness of the hind paws in the control and radiation groups rapidly increased and reached the peak value at 0.5 weeks after surgery. The values in both groups gradually reduced from 0.5 weeks after the surgery. The differences of the hindpaw thickness between the radiation and control groups

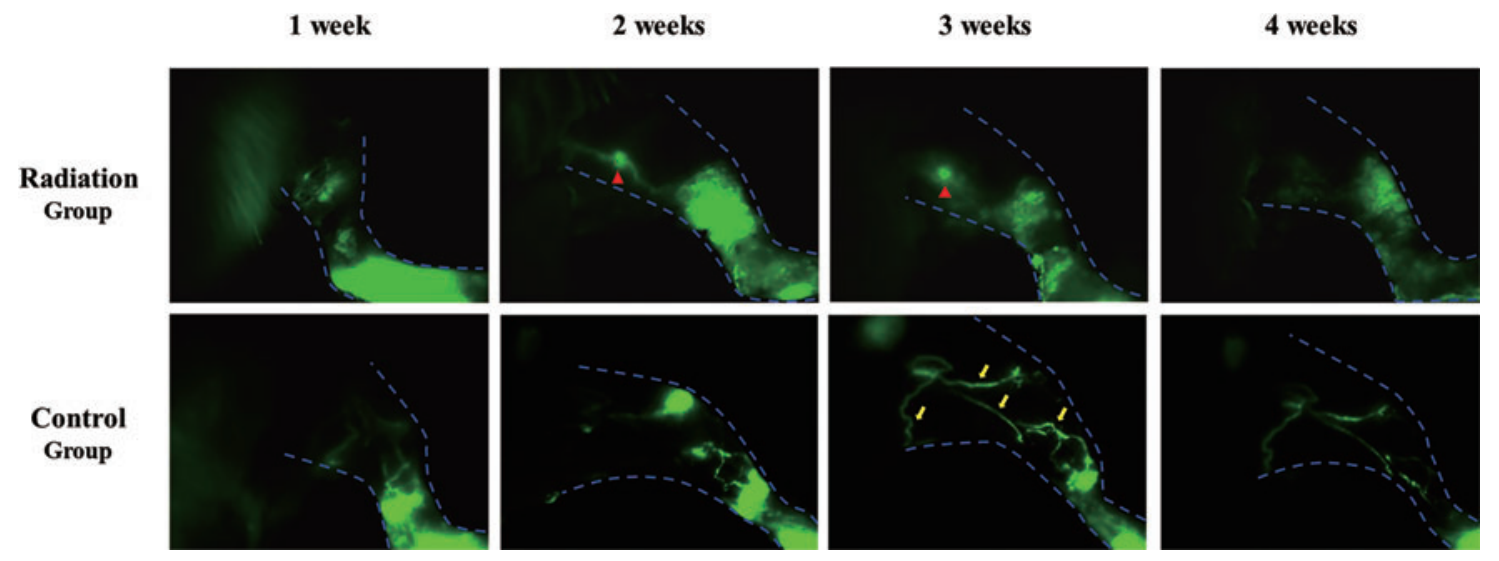

FIG. 3. The fluorescence lymphography shows less regenerated lymphatics and more congestion of tracers in the radiation group at 1, 2, 3, and 4 weeks after surgery. Arrow heads indicate inguinal lymph node; arrows indicate the regenerated lymphatic vessels. Color images are available online. 

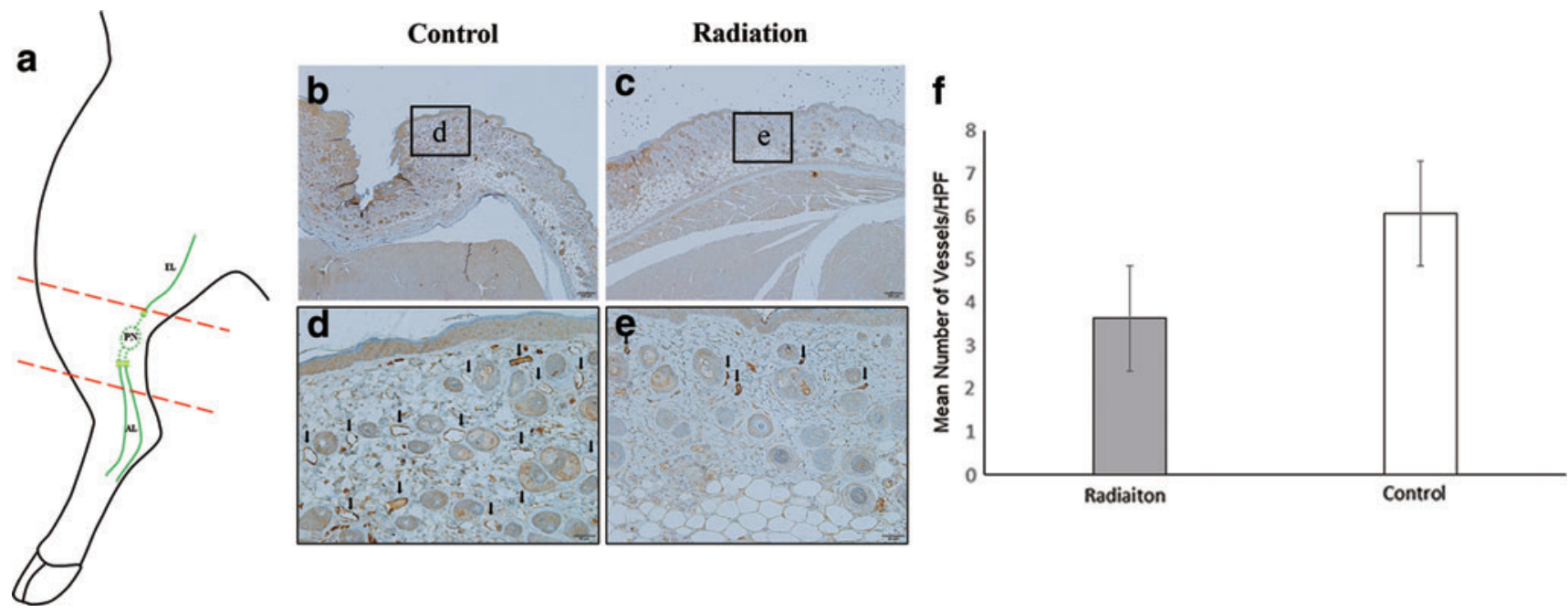

FIG. 4. IHC analysis of dermal lymphatics in the radiation and control groups. (a) Schematic diagram of the surgical area and sample collection. The area between the two parallel dashed lines indicates the range of the sample for IHC staining. The rectangular area indicates the surgical area. (b-e) IHC staining of lymphatics in both groups (anti-LYVE-1 staining, low magnification; $40 \times$ and high magnification; $200 \times$ ). Images were taken from the tissues of $5 \mathrm{~mm}$ distal to the surgery sites [(b) and (c): $40 \times ;(\mathbf{d})$ and (e): $200 \times$ ]. Arrows indicate dermal lymphatic vessels. (f) Dermal lymphatics in each group were quantified. AL, afferent lymphatic; EL, efferent lymphatic; IHC, immunohistochemical; LYVE-1, lymphatic endothelial hyaluronan receptor; PN, popliteal lymph node. Color images are available online.

were statistically significant from 0.5 to 2 weeks $(5.0 \pm 0.2 \mathrm{~mm}$ vs. $4.7 \pm 0.1 \mathrm{~mm}, p=0.026$ at 0.5 weeks; $4.8 \pm 0.2 \mathrm{~mm}$ vs. $4.4 \pm$ $0.1 \mathrm{~mm}, p=0.012$ at 1 week; $4.6 \pm 0.2 \mathrm{~mm}$ vs. $4.2 \pm 0.1 \mathrm{~mm}$, $p=0.032$ at 1.5 weeks; $4.4 \pm 0.2 \mathrm{~mm}$ vs. $4.1 \pm 0.1 \mathrm{~mm}, p=$ 0.046 at 2 weeks) (Fig. 2).

\section{Fluorescence lymphography}

The radiation group showed a lower number of regenerated lymphatics in the operated limb and more congestion of tracers distal to the surgery site at 1, 2, 3, and 4 weeks after surgery. The image in the radiation group obtained at 3 weeks showed that inguinal lymph node occurred because of the lack of regenerated lymphatics (Fig. 3).

\section{IHC staining}

The radiation group showed a lower number of regenerated lymphatics per HPF at the surgery site than the control group $(3.6 \pm 2.4$ vs. $6.1 \pm 2.4 ; p<0.01)$ (Fig. 4$)$.

\section{Discussion}

In this study, we showed that secondary lymphedema was augmented in the radiation group. We found that the number of regenerated lymphatic channels were lower in the radiation group. This finding is supported by histological analysis that demonstrated a lower number of LYVE-1-stained lymphatic vessels in the radiation group. In our study, an improved method was used to create a lymphedema hindlimb model. We blocked the lymphatic components without injecting blue dues. For the previous hindlimb models, ${ }^{11,14,15}$ researchers used various blue dye to identify the lymph nodes and lymphatics. The toxicity of blue dyes caused an inflammatory response, which led to tissue edema. It was not possible to distinguish the tissue edema results from the lymph node excision or toxicity of blue dyes in their studies.
Previous studies have demonstrated the incapability of surgery to create a sustained lymphedema model because the lymphatic endothelial cells' (LECs) proliferation and organization result in spontaneous lymphatic reconnection. Roh et al. ${ }^{11}$ devised a mouse hindlimb lymphedema model by excising a popliteal lymph, a subiliac lymph node, a sciatic lymph node, and cauterization of the femoral lymphatic vessels without radiation. In this lymphedema model, a significant difference in the degree of lymphedema between the surgery and control groups was maintained only for 18 days after the surgery. Radiation therapy has been used as an adjunctive method in the creation of tail and hindlimb lymphedema model. Previous study ${ }^{18}$ demonstrated the effects of radiation therapy on the lymphatic vessels in a tail model. Researchers found that radiation therapy decreases the number of dermal capillary lymphatic vessels and LECs and that a single $15 \mathrm{~Gy}$ dosage is sufficient to cause the loss of lymphatic vessels. Our fluorescence lymphography and histological finding are consistent with their finding. Lymphangiogenesis was hindered in the radiation group compared with that in the control group, which may contribute to the higher lymphedema degree in the radiation group.

However, we found that the duration of secondary lymphedema was not significantly different between the two groups. Apart from lymphatic vessel drainage, interstitial flow (IF) has been indicated, another important route for lymphatic fluid clearance. ${ }^{19,20}$ From the fluorescence image in the radiation group obtained at 3 weeks, we can see that the inguinal lymph node was shown because of the lack of regenerated lymphatics. This finding suggests the tracer mainly passed through the surgical site by interstitial flow rather than regenerated lymphatics, which may result in nonsignificant difference of the lymphedema duration between the two groups. Uzarski et al. ${ }^{21}$ also indicated that the resolution of lymphedema may become more dependent on IF than on lymphangiogenesis in a mouse tail model. 
Lymphangiogenesis has only been considered as a clearance route by most studies using hindlimb models. However, our finding implicates that IF plays an important role in lymphatic fluid clearance. Modified suture technique, such as suturing the skin edges to the underlying muscle or electrocauterizing both edges of the wound, may be valuable for blocking IF. For instance, Oashi et al. ${ }^{14}$ developed a lymphedema mouse hindlimb model. The duration of lymphedema can last several months. To block the dermal backflow, they sutured the skin edges to the underlying muscle with 6-0 nylon sutures leaving a gap of 1-2 mm between skin edges. Similarly, Iwasaki et al. ${ }^{22}$ developed a new hindlimb model by removing both the inguinal and popliteal lymph nodes, followed by silicone splint suture in the inguinal region. The lymphedema degree and duration were significantly increased by their revised surgical method. However, in our study, the ends of the skin wound were directly sutured with 5-0 nylon sutures, which means the dermal backflow reconstructed more quickly by the acceleration of wound healing. Therefore, the lymphedema duration can only last $<3$ weeks. Further studies on the hindlimb lymphedema model should consider both the factors of lymphangiogenesis and interstitial flow.

There are some limitations to this study. First, we did not evaluate the effect of tissue fibrosis on lymphedema. Radiation therapy is known to cause transforming growth factor-beta 1mediated tissue fibrosis, ${ }^{23-25}$ which is another potential mechanism to induce lymphatic stasis. Second, a single 20 Gy radiation was only administered to each mouse, yielding the results of this study. We selected $20 \mathrm{~Gy}$ as the dose of radiation because it was widely used in previous studies. However, we cannot exclude the possibility that the results would be affected by different dose. Considered together, the effect of various dosage radiation on the lymphatic stasis and the mechanism associated with the fibrosis are required to be investigated in the future.

\section{Conclusions}

This study showed that radiation transiently aggravated the lymphedema degree by inhibiting regenerated lymphatics in a mouse hindlimb model. However, it did not prolong the duration of lymphedema because the cutaneous interstitial flow contributes to lymphatic fluid clearance. A sustained hindlimb lymphedema model may be developed by using the combination of lymphatic components incision, radiation, and the method to block the interstitial flow.

\section{Acknowledgment}

This work was supported by the National Research Foundation of Korea (NRF) grant funded by the Korea government (MSIT) (No. 2019R1A2C1009055) and a grant (2017-478) from the Asan Institute for Life Sciences, Asan Medical Center, Seoul, Korea.

\section{Author Disclosure Statement}

The authors have declared no conflict of interests.

\section{References}

1. Ramaiah KD, Ottesen EA. Progress and impact of 13 years of the global programme to eliminate lymphatic filariasis on reducing the burden of filarial disease. PLoS Negl Trop Dis 2014; 8:e3319.
2. Brayton KM, Hirsch AT, O Brien PJ, Cheville A, KaracaMandic P, Rockson SG. Lymphedema prevalence and treatment benefits in cancer: Impact of a therapeutic intervention on health outcomes and costs. PLoS One 2014; 9:e114597.

3. Cormier JN, Askew RL, Mungovan KS, Xing Y, Ross MI, Armer JM. Lymphedema beyond breast cancer: A systematic review and meta-analysis of cancer-related secondary lymphedema. Cancer 2010; 116:5138-5149.

4. Meric F, Buchholz TA, Mirza NQ, et al. Long-term complications associated with breast-conservation surgery and radiotherapy. Ann Surg Oncol 2002; 9:543-549.

5. Warren AG, Brorson H, Borud LJ, Slavin SA. Lymphedema: A comprehensive review. Ann Plast Surg 2007; 59: 464-472.

6. Shallwani SM, Hodgson P, Towers A. Comparisons between cancer-related and noncancer-related lymphedema: An overview of new patients referred to a specialized hospital-based center in Canada. Lymphat Res Biol 2017; 15:64-69.

7. Executive C. The diagnosis and treatment of peripheral lymphedema: 2016 Consensus document of the international society of lymphology. Lymphology 2016; 49:170184.

8. Frueh FS, Gousopoulos E, Rezaeian F, Menger MD, Lindenblatt N, Giovanoli P. Animal models in surgical lymphedema research-A systematic review. J Surg Res 2016; 200:208-220.

9. Rutkowski JM, Moya M, Johannes J, Goldman J, Swartz MA. Secondary lymphedema in the mouse tail: Lymphatic hyperplasia, VEGF-C upregulation, and the protective role of MMP-9. Microvasc Res 2006; 72:161-171.

10. Park HS, Jung IM, Choi GH, Hahn S, Yoo YS, Lee T. Modification of a rodent hindlimb model of secondary lymphedema: Surgical radicality versus radiotherapeutic ablation. Biomed Res Int 2013; 2013:208912.

11. Roh K, Cho S, Park JH, et al. Therapeutic effects of hyaluronidase on acquired lymphedema using a newly developed mouse limb model. Exp Biol Med (Maywood) 2017; 242:584-592.

12. Tammela T, Saaristo A, Holopainen T, et al. Therapeutic differentiation and maturation of lymphatic vessels after lymph node dissection and transplantation. Nat Med 2007; 13:1458-1466.

13. Dai TT, Cui CX, Cao WG, Jiang ZH, Li SL. A refined mouse hind limb model of secondary lymphedema. Int $\mathbf{J}$ Clin Exp Med 2016; 9:7565-7572.

14. Oashi K, Furukawa H, Oyama A, et al. A new model of acquired lymphedema in the mouse hind limb: A preliminary report. Ann Plast Surg 2012; 69:565-568.

15. Jun H, Lee JY, Kim JH, et al. Modified mouse models of chronic secondary lymphedema: Tail and hind limb models. Ann Vasc Surg 2017; 43:288-295.

16. Shin WS, Szuba A, Rockson SG. Animal models for the study of lymphatic insufficiency. Lymphat Res Biol 2003; 1:159-169.

17. Huang JJ, Gardenier JC, Hespe GE, et al. Lymph node transplantation decreases swelling and restores immune responses in a transgenic model of lymphedema. PLoS One 2016; 11:e0168259.

18. Avraham T, Yan A, Zampell JC, et al. Radiation therapy causes loss of dermal lymphatic vessels and interferes with lymphatic function by TGF-beta 1-mediated tissue fibrosis. Am J Physiol-Cell Ph 2010; 299:C589-C605. 
19. Boardman KC, Swartz MA. Interstitial flow as a guide for lymphangiogenesis. Circ Res 2003; 92:801-808.

20. Coffindaffer-Wilson M, Craig MP, Hove JR. Normal interstitial flow is critical for developmental lymphangiogenesis in the zebrafish. Lymphat Res Biol 2011; 9:151158.

21. Uzarski J, Drelles MB, Gibbs SE, et al. The resolution of lymphedema by interstitial flow in the mouse tail skin. Am J Physiol Heart Circ Physiol 2008; 294:H1326-H1334.

22. Iwasaki D, Yamamoto Y, Murao N, Oyama A, Funayama E, Furukawa H. Establishment of an acquired lymphedema model in the mouse hindlimb: Technical refinement and molecular characteristics. Plast Reconstr Surg 2017; 139: $67 \mathrm{e}-78 \mathrm{e}$.

23. Clavin NW, Avraham T, Fernandez J, et al. TGF-beta1 is a negative regulator of lymphatic regeneration during wound repair. Am J Physiol Heart Circ Physiol 2008; 295:H2113$\mathrm{H} 2127$.

24. Oka M, Iwata C, Suzuki HI, et al. Inhibition of endogenous TGF-beta signaling enhances lymphangiogenesis. Blood 2008; 111:4571-4579.

25. Avraham T, Daluvoy S, Zampell J, et al. Blockade of transforming growth factor-betal accelerates lymphatic regeneration during wound repair. Am J Pathol 2010; 177: 3202-3214.

Address correspondence to:

Jae Yong Jeon, MD, PhD

Department of Rehabilitation Medicine Asan Medical Center

University of Ulsan College of Medicine 88, Olympic-ro 43-gil Songpa-gu, Seoul 05505 Republic of Korea

E-mail: jyjeon71@gmail.com

Ho-Young Song, MD, PhD Department of Radiology Asan Medical Center

University of Ulsan College of Medicine 88, Olympic-ro 43-gil Songpa-gu, Seoul 05505 Republic of Korea

E-mail: hysong@amc.seoul.kr 